\title{
SYNTHETIC P-AGB EVOLUTION
}

\author{
LETIZIA STANGHELLINI \\ Osservatorio Astronomico di Bologna, via Zamboni 33, I-40126 Bologna, Italy \\ and \\ ALVIO RENZINI \\ Dipartimento di Astronomia, Università di Bologna, via Zamboni 33, I-40126 Bologna, Italy
}

\begin{abstract}
Extensive Montecarlo simulations of Post-Asymptotic Giant Branch $(P-A G B)$ populations have been constructed, exploring the effects of various assumptions on synthetic $H-R$ diagrams, luminosity functions, and inferred mass distributions. Such assumptions include the IMF, the initial mass-final mass relation, the $A G B$ to $P N$ transition time, the duration of the planetary nebula (PN) stage, etc. We have also investigated how the observational errors in luminosity and temperature propagate into the inferred mass distribution of the P-AGB stars.
\end{abstract}

In recent years many authors have compared theoretical evolutionary tracks for P-AGB stars to observational data of Planetary Nebulae Nuclei (PNNi) in order to infer several properties of these stars (e.g. Schönberner 1981; Tylenda et al. 1991; Stasińska et al. 1991). While several interesting results have been achieved, it became also clear that some of these comparisons may be affected by intrinsic limitations (cf. Acker 1989; Schönberner \& Tylenda 1990) some of which are further explored in this paper. We have developed a versatile method to produce Montecarlo simulations of P-AGB stars based on the evolutionary tracks of Paczyński (1971) and Schönberner (1983). The method is able to produce a synthetic population of PNNi for specified IMF, AGB to PN transition time, and initial mass-final mass relation. Only a very restricted selection of our simulations is presented here, while a more extensive set will be published elsewhere in the near future.

In principle, the mass of $\mathrm{PNNi}$ can be determined from their location in the H-R diagram, making use of P-AGB evolutionary tracks. However, both the luminosity and the effective temperature of $\mathrm{PNNi}$ are affected by systematic and random errors that affect the derived mass distribution. Here we explore the effect of systematic errors in temperature. The temperatures are in general estimated using the Zanstra method, which assumes the nebula to be optically thick to the ionizing radiation. The method gives different results depending on the considered ion; in particular, the H I Zanstra temperature is almost always lower than the $\mathrm{He}$ II one, being the nebulae generally more optically thick to the radiation able to ionize the latter ion. Since in some cases the nebula is thin to He II ionizing radiation too, it appears clear how uncertain these temperature determinations may be. For these reasons, we have constructed two P-AGB stellar populations with completely identical premises, except that in one case we arificially introduce a $30 \%$ systematic error in the effective temperature. We extract synthetic P-AGB populations of 200 objects each, using a Salpeter's IMF, and Weidemann's (1987) initial mass-final mass relation. A $\sim 30 \%$ error in temperature is rather common for the Zanstra method.

Figure 1 shows the effect of the temperature error on the resulting $H-\mathrm{R}$ diagram (left panel) and on the inferred mass distribution (righ panel). One can see how different the two mass distributions are; the average PNN mass is respectively 
$<M>=0.596 M_{\odot}$ for the true distributions and $<M>=0.669 M_{\odot}$ for the observed one. We should nonetheless note that the peak in the mass distributions is stable against effective temperature errors, while only the high mass tail of the distribution is affected. This particular behavior follows from the low mass objects being predominantly on the horizontal ( $L \simeq$ const) part of the tracks, while higher mass PNNi are found predominatly in the fading sections of them. Therefore, temperature errors do not appreciably affect the inferred mass of the former, while do so for the latter ones. With the same line of reasoning we can also easily realize that errors in the PN distance scale will have the opposite effect. We conclude that this method of deriving the mass distribution of PNNi is prone to systematic (and random) errors in the effective temperature of individual objects, in particular for what concerns the high mass tail of the distribution.
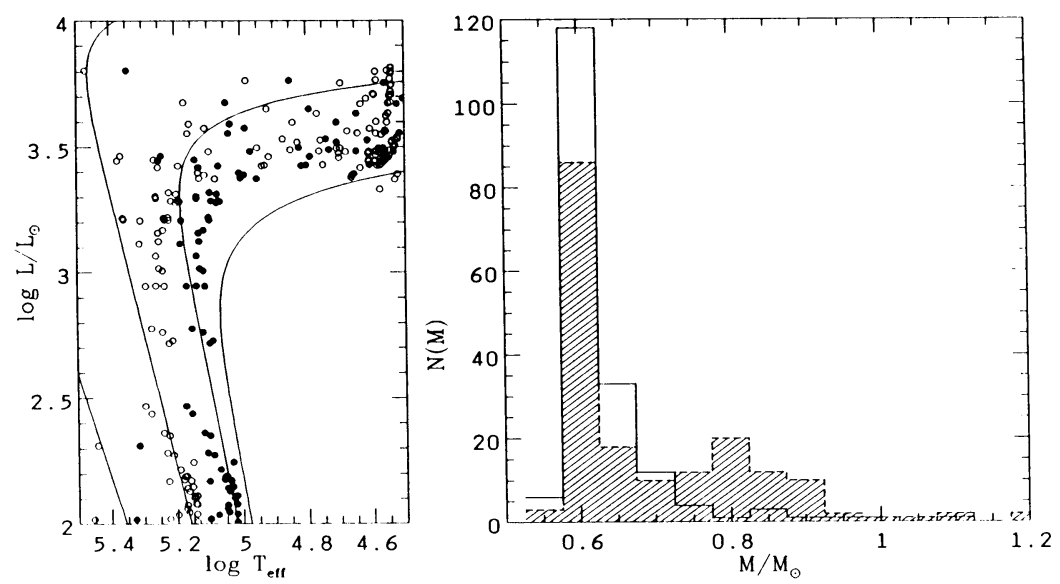

Fig. 1. Left: $P-A G B$ populations on the $H$ - $R$ diagram; filled circles: true distribution; open circles: observed distribution. The tracks correspond to $M / M_{\odot}=$ 0.55,0.6,0.8,1.2. Right: corresponding mass histograms; solid line: input or true distribution; broken line and shaded histogram: derived or observed distribution.

In Figure 2 we show a further example of how systematic errors in the temperature scale can interfere with other aspects of the problem and potentially lead to erroneous conclusions. Figure 2 shows three mass histograms of synthetic populations that differ only for the initial mass-final mass relation. We can see that the bimodality in the mass distribution of Figure 1 referring to the case with temperature error included mimics that shown in Figure 2 when the initial mass-final mass relation adopted by Ciotti et al. (1991) is used instead of that of Weidemann (1987).

The last test concerns the problem of the AGB to PN transition time. It is well known that the planetary nebula formation depends on the delicate interplay between the time scales of the dynamical evolution of the shell and the evolution of the nucleus. The AGB-PN transition time is controlled by the exact value of the envelope mass $\left(M_{\mathrm{e}}^{\mathrm{R}}\right)$ that survives the superwind envelope ejection at the tip of the AGB (Renzini 1989), a quantity that can hardly be theoretically predicted.

Recent observations associated to simple modelling (Käufl, Renzini, \& Stanghellini 
1992) have shown that on average the transition time is of the order of $1000 \mathrm{yrs}$ or more, i.e., a non negligible fraction of the nebular time scale. In Figure 3 we show the effects of the adopted $M_{\mathrm{e}}^{\mathrm{R}}$ on the luminosity function of a systhetic distribution of $\mathrm{PNNi}$, via the implied transition time. The first synthetic distribution is derived under the assumption of a constant residual envelope mass $\left(M_{\mathrm{e}}^{\mathrm{R}}=10^{-3} M_{\odot}\right)$, irrespective of the stellar mass; for the second simulation (following Paczyński 1971) we have adopted a linear $M_{\mathrm{e}}^{\mathrm{R}}(M)$ relation, with $M_{\mathrm{e}}^{\mathrm{R}}=10^{-2}$ and $10^{-3} M_{\odot}$ respectively for $M=1.2$ and $0.6 M_{\odot}$. The resulting luminosity functions are clearly very sensitive to the adopted transition times. We should caution however that for these simulations we have not taken into account the effects of the duration of the PN phase, which is also expected to be a function of the stellar mass. The luminosity function in Figure 3 referes to all P-AGB objects with luminosity down to $\log L=1.5$, which in the case of low mass stars is reached in a time much longer than the PN phase. The effect of the duration of the PN phase will be thoroughly examined in our forthcoming paper, along with other explorations.

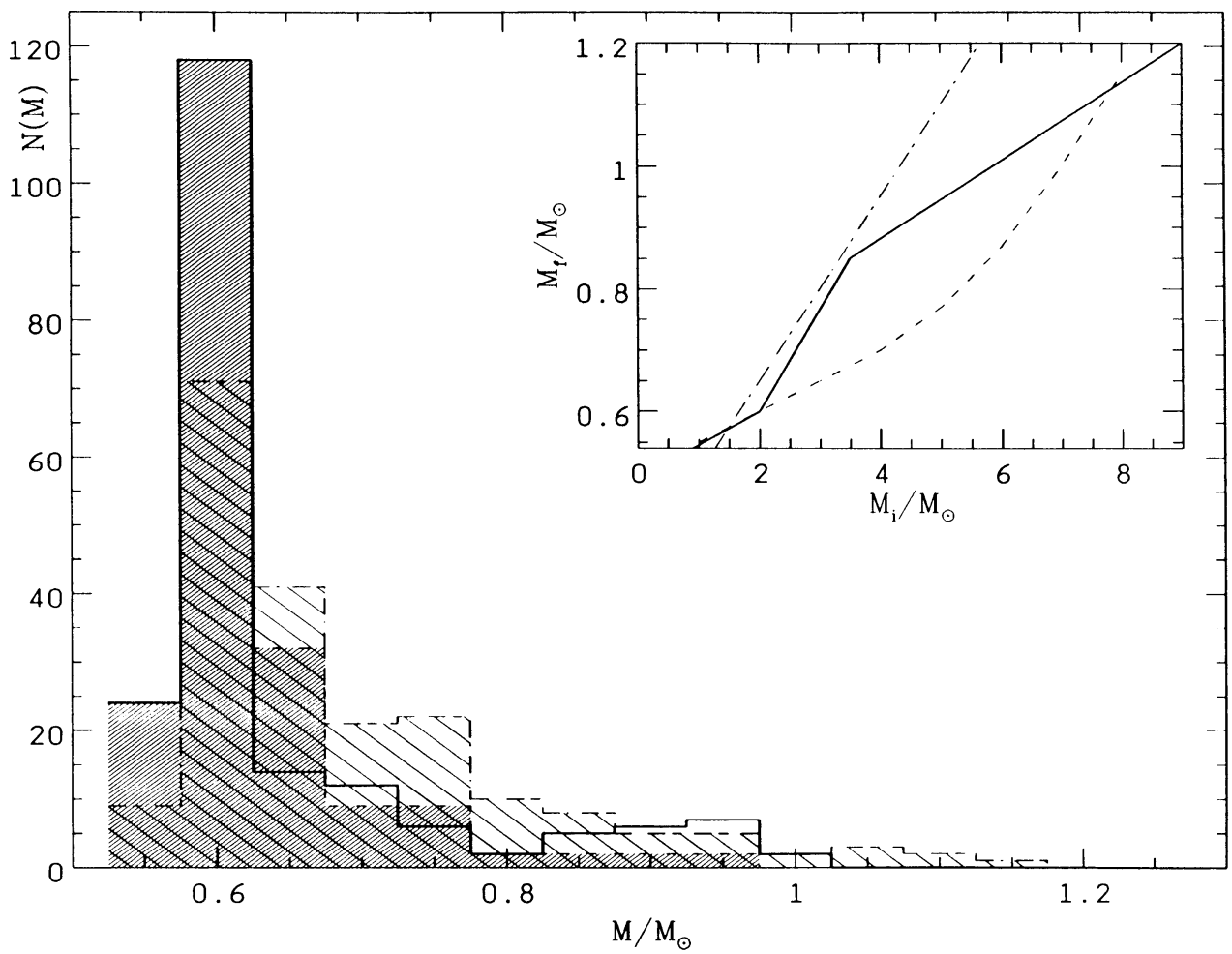

Fig. 2. Effects of different initial mass-final mass relations on the PNN mass distribution. The initial mass-final mass relations (shown in the insert) are from Iben $\&$ Renzini (1983), with $\eta=2$ and $b=1$, dot-dash line and lightly shaded histogram, Ciotti et al. (1991), solid line and unshaded histogram, and Weidemann (1987), dashed line and heavily shaded histogram.

In conclusion, errors in the effective temperature can produce strong variations in 
the inferred mass distribution, and can mask the effect of the initial mass-final mass relation. Also, current uncertainties affecting the AGB to PN transition times can have serious effects on the predicted luminosity function of PNNi.
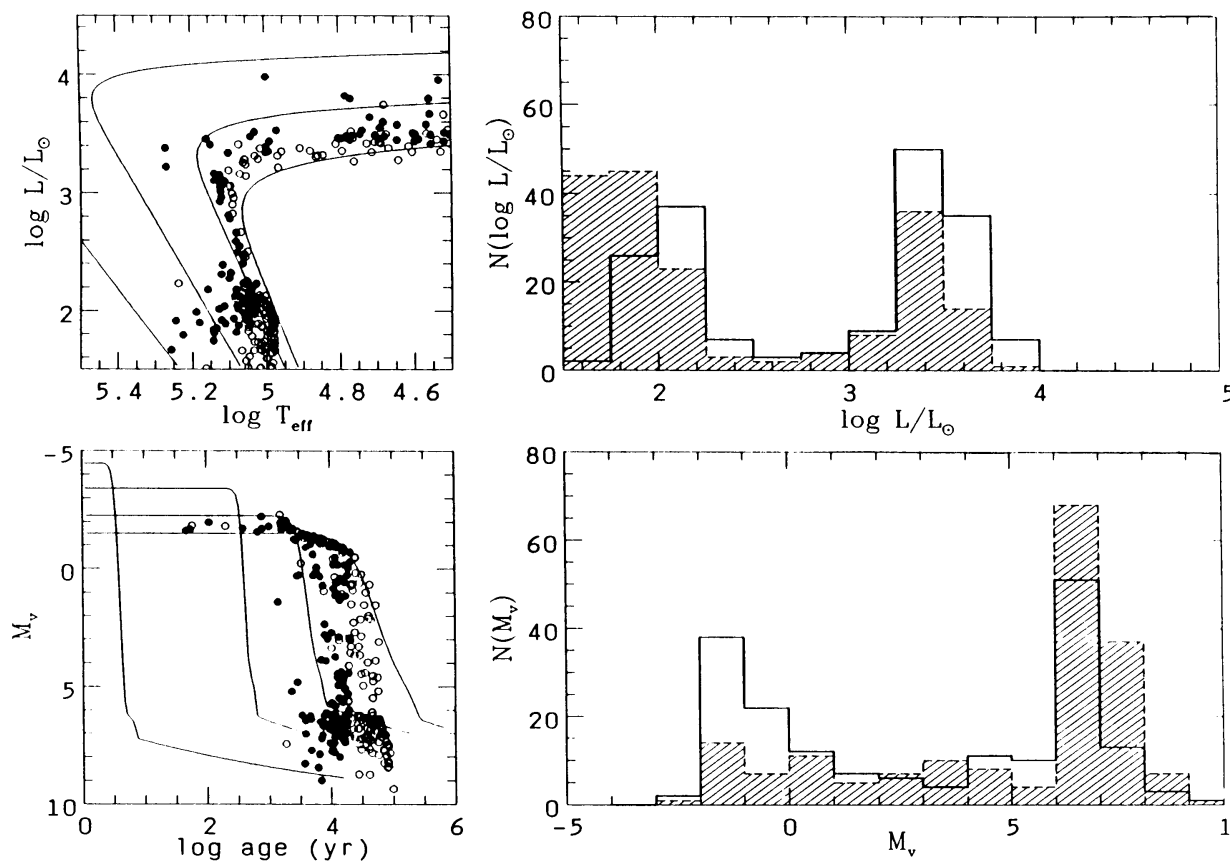

Fig. 3. Transition time effects on luminosity distribution. Left panels: distribution of 200 PNNi on the $H-R$ and $M_{v}-t$ diagrams; solid symbols: case $A$ with $M_{\mathrm{e}}^{\mathrm{R}}=10^{-3} M_{\odot} ;$ open symbols: case $B$ with $M_{\mathrm{e}}^{\mathrm{R}}(M)$. Right panels: corresponding luminosity functions, with solid/unshaded histograms for case $A$, and dashed/shaded histogram for case $B$.

\section{References}

Acker A., 1989, in Planetary Nebulae, ed. A. Acker (Starsbourg Obs.), p. 77 Ciotti, L., D'Ercole, A., Pellegrini, S., \& Renzini, A. 1991, ApJ, 376, 380

Käufl H. U., Renzini A., \& Stanghellini L., 1992, ApJ submitted

Paczyński B., 1971, Acta Astron. 21, 417

Renzini A., 1989, in Planetary Nebulae, ed. S. Torres-Peimbert (Dordrecht: Kluwer), p. 391

Schönberner D., 1981, A\&A 103, 119; 1983, ApJ 272, 708

Schönberner D. \& Tylenda R., 1990, A\&A 234, 439

Stasińska G., et al. 1992, in Stellar Populations of Galaxies, ed. B. Barbuy \& A. Renzini (Dordrecht: Kluwer), p. 492

Tylenda R., Stasińska G., Acker A., \& Stenholm B., 1991, A\&A 246, 221 